Quim. Nova, Vol. 27, No. 1, 84-88, 2004

\title{
ALCÓXIDOS COMO PRECURSORES NA SÍNTESE DE NOVOS MATERIAIS ATRAVÉS DO PROCESSO SOL-GEL
}

\author{
Claudio Airoldi* \\ Instituto de Química, Universidade Estadual de Campinas, CP 6154, 13084-971 Campinas - SP \\ Robson Fernandes de Farias \\ Departamento de Química, Universidade Federal de Roraima, CP 167, 69301-970 Boa Vista - RR
}

Recebido em 17/9/02; aceito em 15/5/03

\begin{abstract}
ALKOXIDE AS PRECURSORS IN THE SYNTHESIS OF NEW MATERIALS THROUGH THE SOL-GEL PROCESS. An overview about the role of alkoxides in the most recent uses of the sol-gel process in the synthesis of new materials is presented. Special attention is focused on the uses of silicon, aluminum, zirconium and titanium alkoxides. This review shows that the alkoxides enable the synthesis of new matrices with controlled surface area, acidity and porosity, as well as some unusual properties. The property associated with the solubility of metal alkoxides opens enormous possibilities of combining them for the synthesis films of powders with a very large range of metal compositions.
\end{abstract}

Keywords: sol-gel; alkoxide; materials.

\section{INTRODUÇÃO}

Nos últimos anos, os profissionais em Química foram aos poucos se familiarizando com uma nova denominação, corriqueiramente utilizada, a fim de destacar o emprego cada vez mais freqüente de novos compostos químicos para fins tecnológicos, que consiste na Química dos Materiais. Aos poucos o poderio da Química se projetou num espaço interdisciplinar, ou mesmo multidisciplinar. A clássica divisão entre as quatro áreas da química, a saber, químicas inorgânica, orgânica, analítica e físico-química, torna-se cada vez menos definida. Tal interpenetração das diferentes sub-áreas faz-se sentir nitidamente na chamada Química supramolecular ${ }^{1,2}$. As inovações que propiciaram o surgimento deste novo campo de pesquisa, consistiram no envolvimento de todos os conhecimentos das áreas antes específicas, na busca de novos compostos, notadamente aqueles com elevado potencial de aplicação tecnológica.

Em se tratando de novos materiais, a primeira impressão que se tem é de que existiria uma larga utilização das clássicas rotas da chamada Química do Estado Sólido que, em princípio, deve estar alicerçada no entendimento da termodinâmica da reação. Desta forma, as condições experimentais podem prever a obtenção de compostos estáveis ou metaestáveis. Para o sucesso de muitas destas operações, fatalmente faz-se necessário o uso de uma metodologia que empregue altas temperaturas na obtenção de cristais, vidros ou materiais cerâmicos. Em caso de baixas temperaturas, pode-se obter agregados cristalinos através da preparação de poliânions a partir de soluções, ou mesmo, em processos sintéticos hidrotérmicos. Em quaisquer casos, as propriedades dos materiais sólidos estão altamente governadas pelas leis termodinâmicas, que se traduzem nos diagramas de fases ${ }^{3}$.

O panorama assim apresentado contrasta com o crescente interesse que vem surgindo através de uma metodologia aplicada à preparação de materiais, que se baseia na polimerização de compostos inorgânicos simples abrindo um campo na ciência dos materiais, que se direciona à Química macromolecular. O sólido pode não ser o mais estável termodinamicamente, mas resulta de um possível con-

*e-mail: airoldi@iqm.unicamp.br trole da reação química ${ }^{4}$ envolvendo, em muitos casos, condições específicas em altas temperaturas.

Contrastando com métodos sintéticos clássicos, destacam-se as sínteses de materiais realizadas à temperatura ambiente, num processo que se popularizou através da expressão francesa "Chimie Douce", cujos princípios se aplicam vastamente dentro da sistemática do processo sol-gel ${ }^{3,5}$. O grande sucesso deste procedimento está relacionado à obtenção de novos materiais que podem desempenhar as mais variadas funções, com especial ênfase no uso em medidas analíticas ${ }^{4,6}$, ou como agentes sequestrantes para metais ${ }^{7}$. Abre-se ainda a possibilidade de preparação de materiais mesoporosos, com várias aplicações, o que vem acompanhado de um enorme conjunto de publicações ${ }^{8,9}$.

Dentre os compostos de constituição simples, utilizados para a preparação de novos materiais via processo sol-gel, destacam-se os alcóxidos, sendo mais particularmente conhecidos os dos elementos silício, alumínio, zircônio e titânio, largamente empregados. Os alcóxidos de silício apresentam uso mais intenso, são os mais estudados, cuja complexa policondensação hidrolítica leva a uma série de reações, que ocorrem simultaneamente, com mecanismos pouco esclarecidos. O mais simples uso deste alcóxido genérico, $\mathrm{Si}(\mathrm{OR})_{4}$, pode ser ilustrado na síntese da sílica, $\mathrm{SiO}_{2}$, que consiste numa rota preparativa com controle cinético da policondensação hidrolítica em torno do átomo de silício, através da substituição dos grupos alcóxidos, pela ação nucleófila da molécula de água, para formar o correspondente álcool ROH. No complexo mecanismo forma-se, intermediariamente, o grupo siloxano $\mathrm{Si}-\mathrm{O}-\mathrm{Si}$, para conduzir à forma final da sílica amorfa. Nesta proposição mecanística o precursor alcóxido passa pela seqüência de oligômero, polímero, colóide, sol, e termina finalmente no sólido ${ }^{3}$.

De um modo geral, a Química de preparação de sólido está intimamente ligada à ciência dos polímeros inorgânicos, no sentido de se sintetizar macromoléculas que, no presente caso, dependem do precursor a ser utilizado no processo sol-gel, tendo em mente o controle cinético da reação. Este aspecto pode conduzir a polímeros que refletem as propriedades macroscópicas como área superficial, porosidade, densidade etc. Muito embora os géis formados sejam descritos como sólidos instáveis, os processos de preparação podem sempre levar à reprodutibilidade do composto final, desde que as 
condições de síntese sejam perfeitamente precisas ${ }^{4}$. Deve-se ainda destacar que, no caso de alcóxidos do tipo $\mathrm{Si}(\mathrm{OR})_{4}$, o tamanho da cadeia (determinada pela natureza do radical $\mathrm{R}$ ) é de fundamental importância para a cinética do processo de hidrólide do alcóxido, verificando-se que, quanto maior a cadeia, mais lento é o processo de hidrólise, o que por sua vez influenciará as propriedades (tamanho de grão e porosidade), por exemplo, do composto formado ${ }^{5}$. Assim, em condições de reação similares, diferentes alcóxidos produzem diferentes materiais. A mesma afirmação é válida para alcóxidos de outros elementos que não o silício.

Este empolgante campo de pesquisa abre uma nova sistemática para a obtenção através de uma seqüência de etapas à temperatura ambiente, estabelecendo uma ponte entre a Química do estado sólido e a molecular, com grande aplicação na formação de matrizes inorgânicas, que podem facilitar, inclusive, a modificação química da superfície. Neste sentido, a divulgação dos recentes avanços na utilização de alcóxidos para a obtenção de novos materiais parece bastante oportuna. O enfoque aqui apresentado será direcionado à Química dos alcóxidos, que apresenta em excelente revisão sobre os aspectos químicos e físicos do processo sol-gel ${ }^{5}$. Os aspectos interdisciplinares destes comportamentos encontram-se esquematizados na Figura 1.

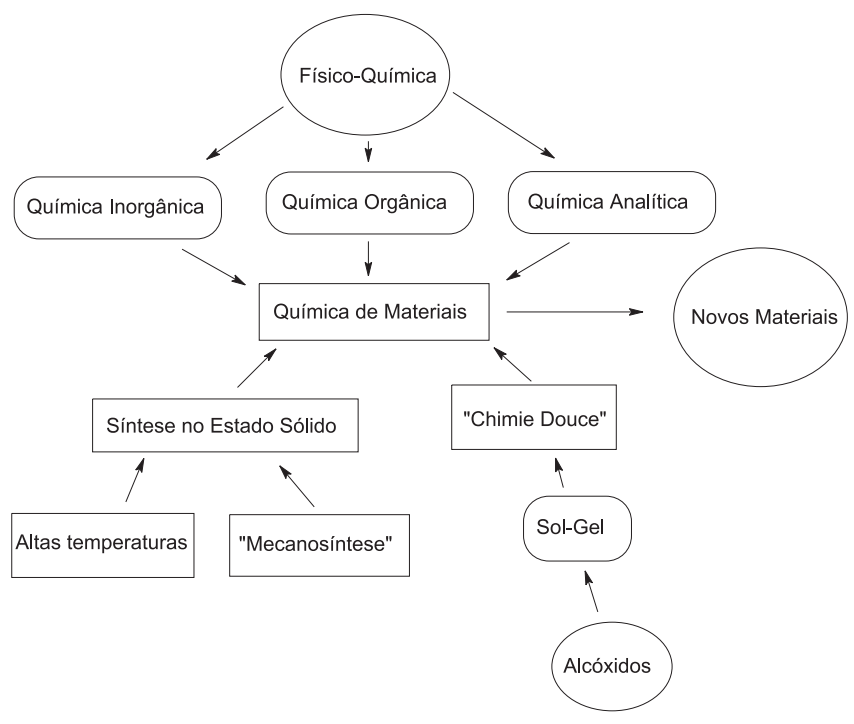

Figura 1. Representação esquemática da natureza interdisciplinar da Química de materiais, e dos papéis desempenhados pelo processo sol-gel e pelos alcóxidos, em particular, na obtenção de novos materiais

A presente revisão apresenta alguns dos mais recentes empregos do processo sol-gel para a síntese de novos materiais, ilustrando a versatilidade desta via sintética. Nos últimos cinco anos, o número de artigos que reportam investigações nas quais o processo sol-gel é empregado pode ser contado à casa dos milhares. Assim sendo, a seleção de exemplos aqui apresentada é apenas uma, dentre muitas outras possíveis. Como critério de seleção, procurou-se escolher relatos ilustrativos, que apresentem aspectos relevantes do emprego sol-gel na síntese de novos materiais.

\section{ALCóXIDOS}

Um alcóxido pode ser entendido como um composto resultante da reação de um haleto metálico com um determinado álcool, em presença de um receptor do ácido formado para deslocar a reação. Outra possibilidade consiste na reação direta do próton do álcool com o metal, com eliminação de hidrogênio gasoso ${ }^{5}$. A preparação de derivados alcóxidos de boro e silício já é descrita desde 1846. Porém, somente por volta de 1950 verificou-se um rápido crescimento na Química preparativa de uma variedade enorme de alcóxidos ${ }^{10}$. Dentre os grupos alcóxidos mais comuns encontram-se

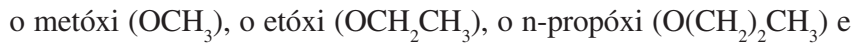
o sec-butóxi $\left(\mathrm{H}_{3} \mathrm{C}(\mathrm{O}) \mathrm{CHCH}_{2} \mathrm{CH}_{3}\right)$. Por outro lado, um dos alcóxidos mais largamente estudados é o tetraetilortossilicato, $\mathrm{Si}\left(\mathrm{OC}_{2} \mathrm{H}_{5}\right)_{4}$, que recebe normalmente a sigla TEOS, cujo largo emprego é direcionado à obtenção de materiais que exigem a presença do silício.

Como é de se esperar, em princípio, qualquer elemento metálico ou semi-metálico pode formar um alcóxido que, devido ao forte efeito de polarização da ligação oxigênio-elemento, sofre facilmente a interação com a molécula de água, numa clássica reação de hidrólise. Do ponto de vista da Química sintética, cuidados especiais são necessários no emprego de condições perfeitamente anidras, para se ter sucesso no isolamento destes tipos de $\operatorname{compostos}^{11}$. Assim, curiosamente, com o avanço da Química na obtenção de produtos de relevância tecnológica, em que se desejava a inclusão de um certo elemento em uma certa matriz, passou-se a explorar a propriedade de hidrólise, derivando uma nova sistemática de pesquisa à temperatura ambiente, através do processo sol-gel, na formação de redes tridimensionais, como bem enfatiza os princípios da "Chimie Douce"3.

Nos dias de hoje, os alcóxidos mais comumente utilizados na síntese de novos materiais são os de silício, alumínio, zircônio e titânio, embora seja possível a síntese de alcóxidos dos mais diversos elementos ${ }^{10}$. Para alcóxidos de arsênio derivados de alcoóis de cadeia normal, por exemplo, verificou-se que a entalpia média da ligação arsênio-oxigênio decresce com o aumento do número de átomos de carbono do radical ligado ao oxigênio ${ }^{12}$.

\section{ALCÓXIDOS E A OBTENÇÃO DE NOVOS MATERIAIS}

\section{Alcóxidos de silício}

Os tetraortoalcoxissilanos deste elemento são representados pela fórmula geral $\mathrm{Si}(\mathrm{OR})_{4}$, sendo mais comuns os compostos formados a partir do metanol (TMOS) e etanol (TEOS). Ambos têm largo emprego na obtenção de novos materiais a partir do processo sol-gel possibilitando, através do polímero tridimensional formado, adicionar o silício na forma de óxido, o qual pode estar acompanhado de outro elemento desejado na mesma matriz. Com esta estrutura formada, pode-se trabalhar quimicamente no sentido de incrustar novos derivados na superfície inorgânica do material sintetizado, através de agentes sililantes adequados, que estão acompanhados de funções químicas, para se atingir uma determinada finalidade na utilização do novo material preparado ${ }^{13-16}$.

Um dos recentes usos dos alcóxidos de silício tem sido na obtenção de sílica lamelar ${ }^{17-25}$, cuja divulgação do tema ${ }^{26}$ mostra o inegável progresso laboratorial, na descoberta de propriedades de hidrólise controlada, para se atingir o objetivo desejado. Neste aspecto é bastante explorado o processo sol-gel, através da chamada rota da diamina neu$\operatorname{tra}^{27}$. Pelo fato de possuir centros básicos, os quais ficam incorporados à matriz inorgânica, as sílicas lamelares assim obtidas, podem ser utilizadas para a extração de metais ${ }^{28}$. Por outro lado, a presença destes elementos metálicos na rede, dispostos no interior da cavidade formada, causa determinadas e significativas alterações à estrutura original, bem como afeta a estabilidade térmica ${ }^{28}$. Desta forma, as reações no estado sólido entre sílica dopadas com nitrato ou sulfato de cobre e brometo de potássio ${ }^{29}$ podem ainda afetar, de forma notável, a estrutura da sílica lamelar obtida via processo sol-gel. Uma representação esquemática da sílica lamelar está apresentada na Figura 2. 


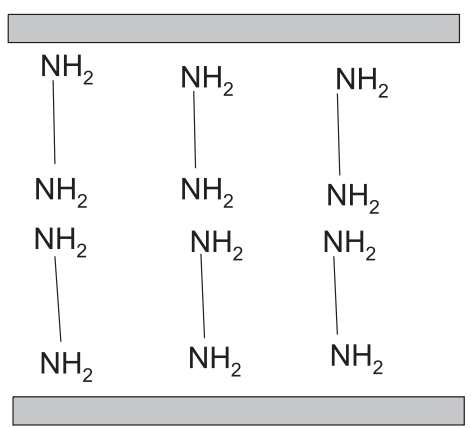

Figura 2. Representação esquemática de sílica lamelar obtida a partir do processo sol-gel, pela reação do tetraortossilicato como precursor e 1,12diaminododecano como molécula modeladora. As barras representam o substrato de $\mathrm{SiO}_{2}$

Controlando-se a cinética dos processos de hidrólise e policondensação ${ }^{30}$ dos alcóxidos de silício mediante controle preciso do $\mathrm{pH}^{31,32}$, pela adição de metais ${ }^{33}$ ou aditivos orgânicos ${ }^{34-38}$ podese variar ou controlar a porosidade da matriz final, bem como a área superficial dos materiais obtidos. Neste procedimento chega-se inclusive a obter sílica ou resinas constituídas por polissiloxanos, sob a forma de belíssimas partículas esféricas ${ }^{39-44}$, enquanto rotas hidrolíticas ${ }^{45}$ ou até mesmo não-hidrolíticas ${ }^{46}$ podem provocar a formação de filmes porosos ${ }^{44}$. Este procedimento mostra uma grande versatilidade nas variações dos aspectos preparativos, quando se explora devidamente os alcóxidos.

A enorme utilização de alcóxidos de silício é retratada como precursora na obtenção de materiais híbridos envolvendo fule$\operatorname{renos}^{47,48}$, assim como a utilização de TMOS e TEOS como aditivos para reforçar a estrutura de polímeros orgânicos ${ }^{49,50}$. Os materiais com propriedades ópticas ${ }^{51,52}$, condutoras $^{53}$ ou catalíticas ${ }^{54-56}$ foram também sintetizados, baseando-se sempre no uso de alcóxidos de silício como precursores. Quando se usou o TEOS em presença de moléculas modeladoras, tornou-se possível a obtenção de materiais com estruturas tubulares ${ }^{57}$. A preparação de partículas de sílica com propriedades específicas para adsorventes cromatográficos ${ }^{58}$, materiais mesoporosos ${ }^{59-61}$ ou redes poliméricas ${ }^{62-64}$ encontram-se também entre os materiais passíveis de síntese mediante hidrólise e policondensação de alcoxissilanos.

Entre as estratégias de síntese mais recentemente utilizadas para a obtenção de materiais híbridos envolvendo silício, está a chamada pilarização $0^{65-68}$, na qual promove-se a hidrólise e polimerização do alcoxissilano no interior de materiais lamelares, tais como fosfatos de zircônio ${ }^{66,67}$, titânio ${ }^{68}$ ou titanatos ${ }^{65}$. Um dos alcoxissilanos mais utilizados é o aminopropiltrietoxissilano, embora resultados recentes ${ }^{69}$ mostrem ser possível efetuar-se a pilarização utilizando-se alcóxidos com cadeias carbônicas consideravelmente maiores.

A preparação de silicatos porosos organicamente modificados foi também reportada ${ }^{70}$, utilizando-se para tal síntese alcóxidos do tipo $\mathrm{RSi}(\mathrm{OEt})_{3}$ com $\mathrm{R}=-\mathrm{CH}_{3},-\mathrm{C}_{2} \mathrm{H}_{5},-\mathrm{C}_{8} \mathrm{H}_{17},-\mathrm{CH}=\mathrm{CH}_{2}$ ou $-\mathrm{C}_{6} \mathrm{H}_{5}$, sendo que o grupo $\mathrm{R}$ exerce influência sobre a porosidade dos materiais calcinados. A organofuncionalização da sílica gel pode também ser conseguida utilizando-se TEOS como precursor, conseguindo-se aumentar a cadeia orgânica ancorada pelo emprego de glutaraldeído ${ }^{71}$.

Além do TEOS e do TMOS, os alcóxidos de silício do tipo $\left(\mathrm{H}_{5} \mathrm{C}_{2} \mathrm{O}\right)_{3} \mathrm{Si}\left(\mathrm{CH}_{2}\right)_{3}-\mathrm{X}$, para $\mathrm{X}=\mathrm{Cl}, \mathrm{NH}_{2}, \mathrm{SH}, \mathrm{Ph}-\mathrm{NH}_{2}$, $\left(\mathrm{CH}_{2}\right)_{2} \mathrm{NH}\left(\mathrm{CH}_{2}\right)_{2} \mathrm{NH}_{2}$ etc, são também muito utilizados, por exemplo, para modificar a superfície da sílica, produzindo superfícies modificadas, com a propriedade de seqüestrar metais em solução ${ }^{72}$. Os complexos suportados assim obtidos podem, por sua vez, ser uti- lizados como catalisadores. Na Figura 3 está representa a interação entre um íon metálico M, e a superfície de sílica organofuncionalizada.

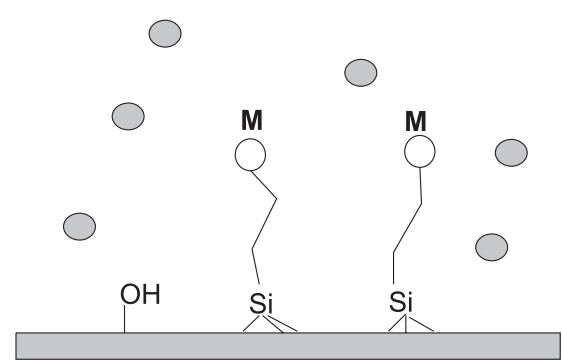

$$
\text { = grupo coordenante } \quad \bigcirc=\text { moléculas do solvente }
$$

Figura 3. Representação esquemática da interação entre sílica organofuncionalizada e cátions metálicos em solução

Os eletrodos para estudos fotoeletroquímicos podem também ser obtidos pelo processo sol-gel, utilizando-se o 3-mercaptopropiltrimetoxissilano, $\left(\mathrm{H}_{3} \mathrm{CO}\right)_{3} \mathrm{Si}\left(\mathrm{CH}_{2}\right)_{3} \mathrm{SH}$ como precursor, a fim de ancorar-se partículas de sulfeto de cádmio na superfície do eletrodo ${ }^{73}$. Tal processo é esquematicamente ilustrado na Figura 4.

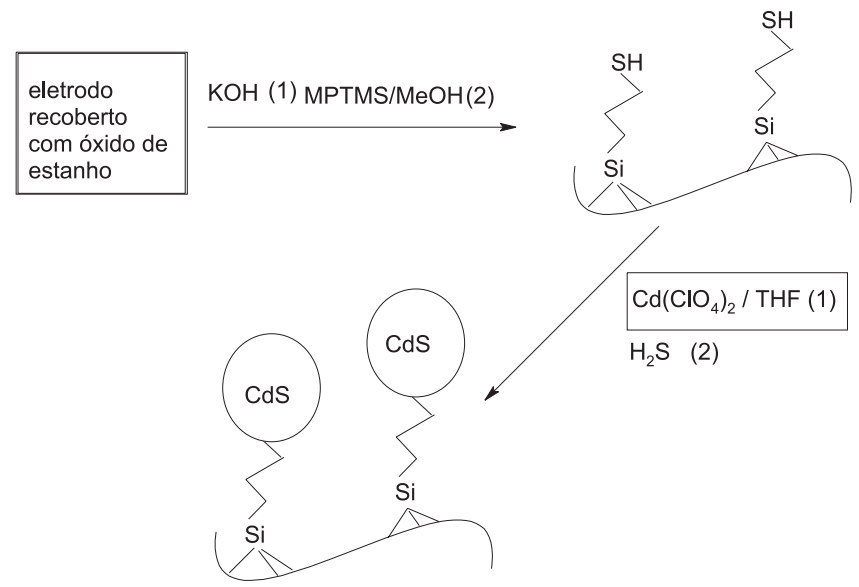

Figura 4. Representação esquemática das etapas envolvidas na modificação de superfície de eletrodo utlizando-se 3-mercaptopropiltrimetoxissilano (MPTMS) como precursor

Como utilização mais recente dos alcóxidos de silício para a obtenção de novos materiais via processo sol gel, pode-se citar a síntese de pós cerâmicos ${ }^{74}$ do tipo $\mathrm{SiO}_{2}-\mathrm{MO},(\mathrm{M}=\mathrm{Zn}, \mathrm{Mn}, \mathrm{Ni}$ e Co), empregando-se sílica lamelar como agente precursor.

\section{Alcóxidos de alumínio, zircônio e titânio}

Houve um considerável aumento no uso de alcóxidos de alumínio, zircônio ou titânio para a obtenção de novos materiais nos últimos anos. Os estudos em grande parte enfocam a síntese de materiais de composição mista, cuja representação dos óxidos formados é simplesmente indicada apenas pela participação dos metais que entraram na formação dos mesmos. Por exemplo, no processo sol-gel envolvendo os alcóxidos para produzir óxidos mistos, os mesmos são denominados por $\mathrm{Zr}-\mathrm{Ti}$, Al-Ti etc ${ }^{75-87}$.

As propriedades físico-químicas de óxidos mistos, como por exemplo $\mathrm{Zr}$-Si, têm despertado enorme interesse pelo fato de que a 
nova composição apresenta elevada estabilidade térmica e química, além de que a superfície dispõe de grupos $\mathrm{OH}$, responsáveis pela elevada acidez. Do ponto de vista experimental, a rota preparativa consiste na hidrólise conjunta da mistura dos alcóxidos, partindo-se do tetra-n-propóxido de zircônio e TEOS como precursores, num típico processo sol-gel, para obter este importante material ${ }^{78}$

Um fato marcante sobre a investigação destes óxidos é a busca de certo teor de cristalinidade que podem apresentar, com o estabelecimento de condições experimentais adequadas. Desta forma, através do uso de um agente modelador incluído no processo sol-gel que, normalmente, se utiliza de uma diamina neutra de cadeia longa, pelo emprego de TEOS, tetrabutóxido de titânio ou zircônio e trisec-butóxido de alumínio, foram obtidos uma série de dióxidos de estruturas lamelares ou hexagonais. Além do mais, estes novos materiais apresentam a habilidade como agente seqüestrante para cátions metálicos dispersos em solução aquosa ${ }^{88}$.

Do ponto de vista estrutural os óxidos mistos de composição 1:1, como por exemplo Zr-Ti, apresentam-se com estrutura hexagonal ${ }^{89}$, quando o mesmo processo sol-gel se utiliza dos precursores tetrabutóxidos. Ressalta-se aqui que estes óxidos mistos quando examinados através da microscopia eletrônica de varredura, apresentaram partículas com morfologia esférica. Porém, os mesmos óxidos mistos, mesmo não sendo obtidos por via hidrolítica, exibem elevada homogeneidade de partículas ${ }^{89}$.

Os precursores tetrabutóxido ou tetrapropóxido de zircônio foram empregados na síntese de materiais híbridos inorgânico-orgânico, após ter sido adicionado ao meio reacional o composto 2 hidroxietilmetacrilato. $\mathrm{O}$ produto final desta preparação conduz a um material homogêneo e transparente, que exibe um comportamento vítreo, com apreciável estabilidade térmica ${ }^{90}$.

Para se sintetizar as amostras lamelares de óxido de zircônio, é imperioso que se empregue a rota da amina neutra, normalmente utiliza-se a 1,12-dodecilamina como molécula modeladora ${ }^{83}$. Explorando-se as propriedades deste material, notou-se que o mesmo apresenta transformações estruturais do tipo lamelar $\rightarrow$ hexagonal $\rightarrow$ cúbica, sendo que a energia de ativação para a transformação lamelar $\rightarrow$ hexagonal é da ordem de $22 \mathrm{~kJ} \mathrm{~mol}^{-1}$.

O óxido de zircônio apresenta-se com elevada dureza e resistência à corrosão e, desta forma, tem sido largamente estudado através de várias possibilidades sintéticas ${ }^{90,91}$. Porém, a melhor condição experimental parece ser aquela em que se emprega a hidrólise e policondensação do tetra-n-propóxido de zircônio, inclusive na preparação de filmes deste óxido, o que propicia o controle sobre a estrutura, morfologia e propriedades mecânicas do óxido resultante.

Num enfoque experimental diferenciado, o processo sol-gel foi efetivado mediante a adição de ácido acético ao meio reacional para a obtenção de óxidos mistos homogêneos do tipo $\mathrm{Si}$-Ti, partindo-se dos agentes precursores tetraisopropóxido de titânio e TEOS ${ }^{92}$. Por outro lado, as partículas esféricas de $\mathrm{TiO}_{2}$ podem ser sintetizadas pela hidrólise e policondensação de tetraetóxido de titânio ${ }^{92-94}$, tendo como mérito o possível controle de área superficial do material obtido.

A fim de ilustrar a influência das condições de síntese sobre os materiais obtidos, cite-se o fato de que o tamanho médio das partículas obtidas é diminuído ao aumentar-se a concentração inicial de água, na preparação de $\mathrm{TiO}_{2}$ a partir do tetraetóxido de titânio ${ }^{94}$. Nessa investigação, assumiu-se que a formação das partículas dava-se por intermédio de um processo homogêneo de nucleação/precipitação, sendo a velocidade de formação das partículas descrito pela equação:

Velocidade $=\kappa\left[\mathrm{Ti}(\mathrm{OR})_{4}\right]\left[\mathrm{H}_{2} \mathrm{O}\right]^{3}$

sendo os respectivos processos de hidrólise e policondensação re- presentados por:

$$
\begin{aligned}
& \mathrm{Ti}(\mathrm{OR})_{4}+3 \mathrm{H}_{3} \mathrm{O} \rightarrow \mathrm{Ti}(\mathrm{OR})(\mathrm{OH})_{3}+3 \mathrm{ROH} \\
& \mathrm{Ti}(\mathrm{OR})(\mathrm{OH})_{3} \rightarrow \mathrm{TiO}_{2} \cdot \mathrm{xH}_{2} \mathrm{O}+(1-\mathrm{x}) \mathrm{H}_{2} \mathrm{O}+\mathrm{ROH}
\end{aligned}
$$

Em um estudo análogo ${ }^{95}$, verificou-se que o fator mais importante na transição anatásio-rutilo é a presença de um terceiro polimorfo, a brooquita, que aumenta a formação da fase rutilo, por fornecer uma rota alternativa para a nucleação. O tamanho das partículas seria o segundo fator mais importante na referida transição. $\mathrm{O}$ efeito do tamanho dos cristalitos deve-se ao fato de que, cristalitos menores tendem a apresentar um maior número de defeitos, em comparação com cristalitos maiores, sendo que a presença de defeitos nos cristalitos facilita a transição estrutural.

Foi recentemente demonstrado ${ }^{95}$ que a transição de fase anatásio $\rightarrow$ rutilo, ambas formas polimórficas do dióxido de titânio, pode ser grandemente influenciada pelo pré-tratamento do óxido amorfo original. Porém, quase que sempre parte-se da hidrólise e policondensação do tetraetilortoetóxido de titânio como uma das rotas de síntese utilizada para a obtenção deste precursor. Verifica-se ainda que, promovendo-se a hidrólise e policondensação do tetrabutóxido de titânio em soluções aquosas saturadas de metais de transição ${ }^{96}$, ou em soluções de dimetilformamida, ou dimetilsulfóxido ${ }^{97}$, obtém-se $\mathrm{TiO}_{2}$ cristalino, com fase anatásio, com diâmetro médio dos cristalitos de 6 ou $11 \mathrm{~nm}$, em vez do óxido amorfo, como é verificado, caso os processos de hidrólise e policondensação sejam conduzidos em água. Dentro desta mesma sistemática de estudo, o tetra-sec-butóxido de alumínio foi também convenientemente utilizado ${ }^{98}$ para a produção de alumina com elevada área superficial, chegando até $575 \mathrm{~m}^{2} \mathrm{~g}^{-1}$, sendo que neste processo preparativo pode-se também controlar o diâmetro e volume de poro do composto final.

\section{Outros alcóxidos}

É possível, em princípio, fazer uso de alcóxido de qualquer elemento e compor uma determinada mistura, podendo ser binário ou de multi componentes. Porém, a título de ilustração de material obtido pelo processo sol-gel, mediante a utilização destes tipos de precursores pode-se citar ainda a preparação do composto $\mathrm{K}_{\mathrm{x}} \mathrm{Ga}_{\mathrm{x}} \mathrm{Sn}_{8-\mathrm{x}} \mathrm{O}_{16}(\mathrm{x}>2)$ obtido a partir de n-propóxido de potássio, trin-butóxido de gálio e tetra-terc-trabutóxido de estanho ${ }^{99}$. A forma cristalina isolada está de acordo com a estrutura característica do tipo holandita, que apresenta propriedades condutoras. Nesta mesma direção destacam-se ainda outros materiais com propriedades condutoras $^{100}$, obtidos via processo sol gel, como $\mathrm{Bi}_{2} \mathrm{Cu}_{0,1} \mathrm{~V}_{0,9} \mathrm{O}_{5,35} \mathrm{e}$ o $\mathrm{Bi}_{2} \mathrm{Nb}_{0,3} \mathrm{~V}_{0,7} \mathrm{O}_{5,5}$.

Os alcóxidos de boro ${ }^{80,101}$, vanádio ${ }^{81}$, estrôncio e bário ${ }^{102}$ são ainda exemplos, até certo ponto pouco explorados, no uso como precursores para a síntese de novos materiais via processo sol-gel.

\section{CONCLUSÕES}

Pelo que se pode perceber consultando alguns dos mais respeitados periódicos dedicados à química de materiais, por exemplo, Chemistry of Materials, Journal of Materials Chemistry etc, verifica-se que o uso do processo-gel, e mais especificamente de alcóxidos, na síntese de novos materiais continua em fase de expansão. Conforme foi apresentado na presente revisão, materiais os mais diversos podem ser preparados mediante o uso de alcóxidos, com as vantagens inerentes ao processo sol-gel, já anteriormente enumeradas. Assim, pesquisas fundamentais que se dediquem à preparação de novos alcóxidos são certamente desejáveis. 


\section{REFERÊNCIAS}

1. Lehn, J. M.; Compreensive Supramolecular Chemistry, BPC Wheatons: Exceter, 1996.

2. Steed, J. W.; Atwood, J.L.; Supramolecular Chemistry, Wiley: New York, 2000 .

3. Corriu, R. J. P.; Eur. J. Inorg. Chem. 2001, 1109.

4. Cerveau, G.; Corriu, R. J. P.; Framery, E.; Chem. Commun. 1999, 2081.

5. Brinker, C. J.; Scherer, G. W.; Sol-Gel Science, Academic Press: San Diego, 1990.

6. Cerveau, G.; Coriu, R. J. P.; Lepeytre, C.F.; Mutin, H.; J. Mater. Chem. 1998, 8, 2707.

7. Murphy, E. F.; Schmid, D.; Burgi, T.; Maciejewski, M.; Baiker, A.; Gunther, D.; Schneider, M.; Chem. Mater. 2001, 13, 1296.

8. Corma, A.; Chem. Rev. 1997, 6, 2373.

9. Di Renzo, F.; Cambon, H.; Dutrartre, R.; Microporous Mesoporous Mater. 1997, 10, 283.

10. Bradley, D. C.; Mehrotra, R. C.; Gaur, D. P.; Metal Alkoxides, Academic Press, London, 1978.

11. Livage, J.; Henry, M.; Sanchez, C.; Prog. Solid State Chem. 1998, 18, 259.

12. Souza, A.G. de; Brasilino, M. G. A.; Airoldi, C.; J. Chem. Thermodyn. 1996, 28, 1359.

13. Prado, A. G. S.; Airoldi, C.; Green Chem. 2002, 4, 288.

14. Airoldi, C.; Arakaki, L. N. H.; J. Colloid Interface Sci. 2002, 249, 1.

15. Silva, C. R.; Bachmann, S.; Schefer, R.R.; Albert, K.; Jardim, I. C. S. F.; Airoldi C.; J. Chromatogr., A 2002, 948, 85.

16. Fonseca, M. G.; Airoldi, C.; Mater. Res. Bull. 2001, 36, 277.

17. Hillhouse, H. W.; Okubo, T.; van Egmond, J. W.; Tsapatsis, M.; Chem. Mater. 1997, 9, 1505.

18. He, J.; Tse, J. S.; Klug, D. D.; Preston, K. F.; J. Mater. Chem. 1998, 8, 705 .

19. Ogawa, M.; Igarashi, T.; Kuroda, K.; Chem. Mater. 1998, 10, 1382.

20. Ogawa, M.; Igarashi, T.; Kuroda, K.; Bull. Chem. Soc. Jpn. 1997, 70, 2833.

21. Zhou, H. S.; Sasabe, H.; Honma, I.; J. Mater. Chem. 1998, 8, 515.

22. Ogawa, M.; Langmuir 1997, 13, 1853.

23. Lim, M. H.; Blanford, C. F.; Stein, A.; Chem. Mater. 1998, 10, 467.

24. Krishnamoorti, R.; Vaia, R. A.; Giannelis, E. P.; Chem. Mater. 1996, 8, 1728 .

25. de Farias, R. F.; Mattínez, L; Airoldi, C.; J. Non-Cryst. Solids 2000, 276, 56.

26. de Farias, R. F.; Airoldi, C.; Quim. Nova 2000, 23, 88.

27. de Farias, R. F.; An. Assoc. Bras. Quim. 1999, 48, 112.

28. de Farias, R. F.; Airoldi, C.; J. Solid State Chem. 2000, 149, 113

29. de Farias, R. F.; Airoldi, C.; J. Non-Cryst. Solids 2000, 261, 181.

30. Assink, R. A.; Kay, B. D.; Colloids Surf., A 1993, 74, 1.

31. Silva, C. R. da; Airoldi, C.; J. Collolid. Interface Sci. 1997, 195, 381.

32. Cihlár, J.; Colloids Surf., A 1993, 70, 239.

33. Öye, G.; Libnau, F. O.; Sjöblom, J.; Friberg, S. E.; Colloids Surf., A 1997, $123,329$.

34. Wen, J.; Dhandapani, B.; Oyama, S. T.; Wilkes, G. L.; Chem. Mater. 1997, 9, 1968

35. Velev, O. D.; Jede, T. A.; Lobo, R. F.; Lenhoff, A. M.; Chem. Mater. 1998, 10, 3597.

36. Zehl, G.; Bischoff, S.; Mizukami, F.; Isutzu, H.; Bartoszek, M.; Jancke, H.; Lücke, B.; Maeda, K.; J. Mater. Chem. 1995, 5, 1893

37. Carr, S. W.; Courtney, L.; Sullivan, A. C.; Chem. Mater. 1997, 9, 1751.

38. Hüsing, N.; Schubert, U.; Misof, K.; Fratzl, P.; Chem. Mater. 1998, 10, 3024

39. Qi, L.; Ma, J.; Cheng, H.; Zhao, Z.; Chem. Mater. 1998, 10, 1623.

40. Yang, H.; Vovk, G.; Coombs, N.; Sokolov, I.; Ozin, G. A.; J. Mater. Chem. 1998, 8, 743 .

41. Huo, Q.; Feng, J.; Schüth, F.; Stucky, G.D.; Chem. Mater. 1997, 9, 14.

42. Izutsu, H.; Mizukami, F.; Nair, P. K.; Kiyozumi, Y.; Maeda, K.; J. Mater. Chem. 1997, 7, 767

43. Alder, K. I.; Sherrington, D. C.; Chem. Commun. 1998, 131

44. Mezza, P.; Phalippou, J.; Sempere, R.; J. Non-Cryst. Solids 1999, 243, 75.

45. Rankin, S. E.; Macosko, C. W.; McCormick, A.V.; Chem. Mater. 1998, 10, 2037.

46. Hay, J.; Porter, D.; Raval, H.; Chem. Commun. 1999, 81.

47. Kraus, A.; Scneider, M.; Gügel, A.; Mülen, K.; J. Mater. Chem. 1997, 7, 763.
48. Signorini, R.; Zerbeto, M.; Meneghetti, M.; Bozio, R.; Maggini, M.; De Faveri, C.; Prato, M.; Scorrano, G.; Chem. Commun. 1996, 1891.

49. Ikeda, Y.; Tanaka, A.; Kohjiya, S.; J. Mater. Chem. 1997, 7, 1497.

50. Ahmad, Z.; Sarwar, M. I.; Mark, J. E.; J. Mater. Chem. 1997, 7, 259.

51. Sung, P.-H.; Hsu, T.-F.; Ding, Y.-H.; Wu, A. Y.; Chem. Mater. 1998, 10, 1642

52. Lee, S.-K.; Okura, I.; Analyst 1997, 122, 81.

53. Fujinami, T.; Tokimuni, A.; Mehta, M. A.; Shriver, D. F.; Rawsky, G. C.; Chem. Mater. 1997, 9, 2236.

54. Lim, M. H.; Blanford, C. F.; Stein, A.; Chem. Mater. 1998, 10, 467.

55. Raimondi, M. E.; Marchese, L.; Giannoti, E.; Maschmeyer, T.; Seddon, J. M.; Collucia, S.; Chem. Commun. 1999, 87.

56. Rhijn, W. M. V.; De Vos, D. E.; Sels, B. F.; Bossaert, W. D.; Jacobs, P. A.; Chem. Commun. 1998, 317.

57. Trau, M.; Yao, N.; Kim, E.; Xia, Y.; Whitesides, G. M.; Aksay, I. A.; Nature 1997, 390, 674

58. Esquena, J.; Pons, R.; Azemar, N.; Caelles, J.; Solans, C.; Colloids Surf., A 1997, 123, 575 .

59. Richer, R.; Mercier, L.; Chem. Commun. 1998, 1775.

60. Schacht, S.; Huo, Q.; Martin-Voigt, I. G.; Stucky, G. D.; Schüt, F.; Science 1996, 273, 768 .

61. Zhao, D.; Yang, P.; Margolese, D. I.; Chmelka, B. F.; Stucky, G. D.; Chem. Commun. 1998, 2499

62. Wang, X.; Dou, T.; Xiao, Y; Chem. Commun. 1998, 1035.

63. Guo, L.; Hyeon-Lee, J.; Beaucage, G.; J. Non-Cryst. Solids 1999, 243, 61. 64. Crivello, J. V.; Mao, Z.; Chem. Mater. 1997, 9, 1554

65. Shangguan, W.; Inoue, K.; Yoshida, A.; Chem. Commun. 1998, 779.

66. Cassagneau, T.; Jones, D. J.; Rozière, J.; J. Phys. Chem. 1993, 97, 8679.

67. Li, L.; Liu, X.; Ge, Y.; Li, L.; Klinowski, J.; J. Phys. Chem. 1991, 95, 5910.

68. Jiao, X.; Chen, D.; Pang, W.; Xu, R.; Yue, Y.; J. Mater. Chem. 1998, 8, 2831.

69. de Farias, R. F.; Nunes, L. M.; Airoldi, C., trabalho não publicado.

70. Babonneau, F.; Leite, L.; Fontlupt, S.; J. Mater. Chem. 1999, 9, 175.

71. Cestari, A. R.; Airoldi, C.; Langmuir 1997, 13, 2681

72. Airoldi, C.; de Farias, R. F.; Quim. Nova 2000, 23, 496.

73. Drouard, S.; Hickey, S. G.; Riley, D. J.; Chem. Commun. 1999, 67.

74. de Farias, R. F. J. Non-Cryst. Solids 2001, 288, 218

75. Curran, M.; Gedris, T. E.; Stiegman, A. E.; Chem. Mater. 1998, 10, 1604

76. Yin, S.; Inoue, Y.; Uchida, S.; Fujishiro, Y.; Sato, T.; J. Mater. Res. 1998, 13,844 .

77. Yanagisawa, K.; Yamamoto, Y.; Feng, Q.; Yamasaki, N.; J. Mater. Res. 1998, 13,825 .

78. Zhan, Z.; Zeng, H. C.; J. Non-Cryst. Solids 1999, 243, 26.

79. Colomban, P.; J. Mater. Res. 1998, 13, 803.

80. On, D. T.; Kapoor, M. P.; Kaliaguine, S.; Chem. Commun. 1996, 1161.

81. Curran, M. D.; Pooré, D. D.; Stiegman, A. E.; Chem. Mater. 1998, 10, 3156.

82. Kohn, S.; Jansen, M.; J. Mater. Chem. 1998, 8, 1593.

83. Lotero, E.; Vu, D.; Nguyen, C.; Wagner, J.; Larsen, G.; Chem. Mater. 1998, $10,3756$.

84. Kodakari, N.; Tomita, K.; Iwata, K.; Katada, N.; Niwa, M.; Langmuir 1998 14, 4623.

85. Camblor, M. A.; Corma, A.; Valencia, S.; J. Mater. Chem. 1998, 8, 2137 86. Collins, D. E.; Bowman, K. J.; J. Mater. Res. 1998, 13, 1230

87. Peterson, M. J.; Peterson, P. J. K.; Ben-Nissan, B.; J. Mater. Res. 1998, 13,388

88. de Farias, R. F.; Airoldi, C.; J. Solid State Chem. 2001, 158, 134

89. de Farias, R. F.; Airoldi, C.; J. Colloid Interface Sci. 1999, 220, 255.

90. Andrianainarivelo, M.; Corriu, R. J. P.; Leclercq, D.; Mutin, P. H.; Vioux, A.; J. Mater. Chem. 1997, 7, 279.

91. Di Maggio, R.; Fambri, L.; Guerriero, A.; Chem. Mater. 1998, 10, 1777.

92. Neeraj; J.; Rao, C. N. R.; J. Mater. Chem. 1998, 8, 1631.

93. Barringer, E. A.; Bowen, H. K.; Langmuir 1985, 1, 414

94. Barringer, E. A.; Bowen, H. K.; Langmuir 1985, 1, 420.

95. Ovenstone, J.; Yanagisawa, K.; Chem. Mater. 1999, 11, 2770.

96. de Farias, R. F.; J. Colloid Interface Sci. 2001, 239, 584.

97. de Farias, R. F.; Ouim. Nova 2002, 25, 1027.

98. Suh, D. J.; Kim, K.-L.; Chem. Mater. 1997, 9, 1903.

99. Fujimoto, K.; Watanabe, M.; Mori, T.; Ito, S.; J. Mater. Res. 1998, 13, 926.

100. Pell, J. W.; Delak, K. M.; Loye, H.-C. zur; Chem. Mater. 1998, 10, 1764.

101. Ichinose, I.; Senzu, H.; Kunitake, T.; Chem. Mater. 1997, 9, 1296.

102. Davies, H. O.; Jones, A. C.; Leedham, T. J.; O'Brien, P.; White, A. J. P.; Willimas, D. J.; J. Mater. Chem. 1998, 8, 2315. 